\title{
Effect of the Norepinephrine Transporter (NET) Inhibition on $\mu$-Opioid Receptor (MOR)-Induced Anti-nociception in a Bone Cancer Pain Model
}

\author{
Hiroko Ono ${ }^{1}$, Atsushi Nakamura ${ }^{1}$, Tomoe Kanbara ${ }^{1}$, Kazuhisa Minami $^{1}$, Shunji Shinohara ${ }^{1}$, \\ Gaku Sakaguchi ${ }^{1}$, and Toshiyuki Kanemasa ${ }^{1, *}$ \\ ${ }^{1}$ Pain \& Neurology, Medicinal Research Laboratories, Shionogi \& Co., Ltd., \\ 1-1, 3-chome, Futaba-cho, Toyonaka, Osaka 561-0825, Japan
}

Received April 2, 2014; Accepted April 30, 2014

\begin{abstract}
Although norepinephrine transporter (NET) inhibition has an additional effect on $\mu$ opioid receptor (MOR)-mediated anti-nociception in inflammatory and neuropathic pain, its effect on cancer pain is not well characterized. We investigated the additional effect of NET inhibition on MOR activation using a mouse femur bone cancer (FBC) pain model by comparing the antinociceptive effect of the dual-acting opioids tramadol and tapentadol and the clinically used MOR-targeted opioids oxycodone and morphine. The anti-nociceptive effects of subcutaneously administered opioids were assessed using the von-Frey filament test. Oxycodone $(1-10 \mathrm{mg} / \mathrm{kg})$ and morphine $(5-50 \mathrm{mg} / \mathrm{kg})$ dose-dependently exhibited potent anti-nociceptive effects, whereas tramadol $(10-56 \mathrm{mg} / \mathrm{kg})$ and tapentadol $(10-30 \mathrm{mg} / \mathrm{kg})$ exhibited partial effects. Rota-rod analyses of tapentadol at a higher dose $(>30 \mathrm{mg} / \mathrm{kg})$ showed a significant decrease in motor coordination, which was partially recovered by pretreatment with MOR or $\alpha_{1}$-adrenoceptor antagonists. The partial anti-nociceptive effect of tapentadol $(30 \mathrm{mg} / \mathrm{kg})$ was completely suppressed by a MOR antagonist, but not by $\alpha_{1}$ - or $\alpha_{2}$-adrenoceptor antagonists, suggesting that neither $\alpha_{1}$-adrenoceptor- nor $\alpha_{2}$-adrenoceptor-mediated pathways are involved in anti-nociception in the FBC model. We conclude that addition of NET inhibition does not contribute to MORmediated anti-nociception in bone cancer pain.
\end{abstract}

Keywords: femur bone cancer pain, norepinephrine transporter, oxycodone, tapentadol, $\mu$-opioid receptor (MOR)

\section{Introduction}

Opioid analgesics are often prescribed to cancer patients based on the World Health Organization (WHO) three-step ladder for pain relief. Clinically used opioids, which selectively act on $\mu$-opioid receptors (MOR), are natural (morphine), semi-synthetic (oxycodone), or synthetic (fentanyl). Dual-acting opioids such as tramadol and tapentadol that have MOR agonist activity and inhibit monoamine transporters are also used clinically to control pain. Although the $O$-desmethylmetabolite [M1] of tramadol and tapentadol show 10-fold lower affinity for MOR than that of oxycodone and

*Corresponding author. toshiyuki.kanemasa@shionogi.co.jp Published online in J-STAGE on June 25, 2014

doi: 10.1254/jphs.14081FP morphine, their combined unique profiles of serotonin/ norepinephrine (NE) transporter inhibition (tramadol) or NE transporter (NET) inhibition (tapentadol) in a single molecule additively or synergistically induce anti-nociceptive effects, along with MOR activation $(1-3)$. Since many opioid-analgesics are available for relieving severe pain, it is important to understand the individual pharmacological characteristics and differences to relieve pain efficiently.

Cancer is one of the most common disorders associated with severe pain, which is reported in $30 \%-50 \%$ of patients with all types of cancer $(4,5)$. In the clinical phase, pain is caused by the cancer itself, progression of cancer, anticancer agents, radiotherapy, and other effects. The underlying pathophysiological mechanism of bone cancer pain is complex. Bone cancer pain is caused by cancer itself and is associated with serious severe pain 
$>7$ on the $0-10$ numerical rating scale (NRS-11) of pain intensity (6). Bone destruction and fracture in bone cancer induces nociceptive and inflammatory pain. In addition, bone metastasis and bone invasion injures peripheral nerves, which induce neuropathic pain.

Femur bone cancer (FBC) is useful because it closely mimics clinical features of human bone cancer pain $(7-9)$. The FBC model is produced by injecting osteolytic sarcoma cells into the intra-medullary space of the mouse left distal femur. Tumor cell growth is correlatively observed with several pain-related behaviors, such as on-going pain, ambulatory pain, and allodynia, considered to be complex pain, followed by neuropathic, nociceptive, and inflammatory pain (10). Using the FBC animal model, we reported previously that oxycodone inhibits all of the on-going, ambulatory, and allodynia pain related behaviors over a similar dose range, whereas morphine and fentanyl have less effect on ambulatory pain (10).

Tramadol and tapentadol show potent anti-nociception for mechanical-allodynia induced by diabetic polyneuropathy (DPN) or osteoarthritic (OA)-induced pain via additive or synergistic NET inhibition and MOR activation $(3,11,12)$, although their effects on bone cancer pain have not been defined. Using the FBC model, we examined potency of the NET-inhibiting function of dual-acting opioids along with MOR activation by comparing their anti-nocicepive effects with those of MOR-selective opioids (oxycodone and morphine).

\section{Materials and Methods}

\section{Animals}

Male $\mathrm{C} 3 \mathrm{H} / \mathrm{HeN}$ mice $(18-23 \mathrm{~g}$, 5-week-old; CLEA Japan, Tokyo) were used for the FBC pain model. Male Sprague-Dawley (SD) rats (150-180 g, 5-week-old; CLEA Japan) were used for the partial sciatic nerve ligation (pSNL) model. Male SD rats $(160-180 \mathrm{~g}$, 6-week-old; Charles River Laboratories Japan, Kanagawa) were used for the oxaliplatin-induced peripheral neuropathy model. Animals were housed in a room maintained at $23^{\circ} \mathrm{C} \pm 1^{\circ} \mathrm{C}$ under a 12-h light/dark cycle and allowed access to water and food ad libitum. All animal procedures were approved by the Animal Care and Use Committee of Shionogi Research Laboratories, Osaka, in agreement with internal guidelines for animal experiments and in adherence to the ethics policy of Shionogi \& Co., Ltd. (Osaka).

\section{Drugs}

Oxycodone hydrochloride and morphine hydrochloride were obtained from Shionogi \& Co., Ltd. Tramadol hydrochloride was from AK Scientific Inc. (Union City,
CA, USA). Tapentadol hydrochloride was from Haoyuan Chemexpress Co., Ltd. (Shanghai, China). The MORselective antagonist $\beta$-FNA was purchased from Tocris Bioscience (Bristol, UK). The $\alpha_{2}$-adrenoceptor antagonist yohimbine and $\alpha_{1}$-adrenoceptor antagonist prazosin were from Sigma-Aldrich (Tokyo). Oxaliplatin was purchased from Wako Pure Chemical Industries, Ltd. (Osaka). Oxaliplatin was dissolved in a $5 \%$ glucose solution (Otsuka Pharmaceutical Co., Inc., Tokyo), and all other drugs were dissolved in $0.9 \%$ physiological saline (Otsuka Pharmaceutical Co., Inc.). Yohimbine, prazosin, and $\beta$-FNA were administrated at 5-min, 30-min, and 24-h before administration of opioids, respectively, as described previously $(13-15)$. The effects of oxycodone and tapentadol were examined based on the timing of their maximum potency.

\section{FBC model}

NCTC 2472 tumor cells (American Type Culture Collection, Manassas, VA, USA) were maintained in Dulbecco's Modified Eagle's Medium (DMEM; Invitrogen, Carlsbad, CA, USA), supplemented with $10 \%$ fetal bovine serum (Invitrogen), 100 units $/ \mathrm{ml}$ penicillin, and $100 \mu \mathrm{g} / \mathrm{ml}$ streptomycin (Invitrogen), and cultured at $37^{\circ} \mathrm{C} \pm 0.2^{\circ} \mathrm{C}$ in a humidified atmosphere of $5 \% \mathrm{CO}_{2}$. To prepare the FBC model, NCTC 2472 tumor cells were injected as described previously $(8,10)$. Briefly, $\mathrm{C} 3 \mathrm{H} / \mathrm{HeN}$ mice were anesthetized with $3 \%$ isoflurane, and left knee arthrotomy performed. Tumor cells $\left(1 \times 10^{5}\right.$ in $5 \mu$ l Hank's balanced salt solution) were injected directly into the medullary cavity of the distal femur, and the hole drilled in the bone was closed with resin cement (ADFA, Kyoto). In the sham group, $5 \mu$ l of Hank's balanced salt solution was injected instead of the tumor cells in the same manner. The effects of opioids were assessed 14 days after tumor implantation, which is the optimal time for evaluation of allodynia in this model (10). Allodynia-like behavior was recognized as ipsilateral paw withdrawal in response to tactile stimuli using a series of von-Frey monofilaments (pressure: 0.008, 0.02, $0.04,0.07,0.16,0.4,0.6$, and $1 \mathrm{~g}$ ). The up-down method of the von-Frey monofilament test was used as described previously (10).

\section{pSNL model}

Rats were anesthetized with 3\% isoflurane, and partial sciatic nerve injury was produced by tying a tight ligature with 4-0 nylon monofilament (Natsume Seisakusyo, Tokyo) around approximately one half of the diameter of the sciatic nerve located on the left ipsilateral side, as described previously $(16,17)$. The sham operation was performed on the right contralateral side of the same animals by exposing the nerve without ligation. 
Drug effects were assessed 14 - 16 days after the operation. The mechanical paw withdrawal threshold was evaluated using von-Frey monofilaments at the ipsilateral side (pressure: 0.4, 0.6, 1, 1.4, 2, 4, 6, 8, 10, 15, $26 \mathrm{~g}$ ). Rats exhibiting thresholds of $8-15 \mathrm{~g}$ on the contralateral side and $0.6-2 \mathrm{~g}$ on the ipsilateral side before drug administration were used.

\section{Oxaliplatin model}

Rat oxaliplatin-induced peripheral neuropathy was induced as described previously (18). Briefly, oxaliplatin $(2 \mathrm{mg} / \mathrm{kg}$ ) or vehicle was injected intraperitoneally (i.p.) twice a week for 4 weeks (days 1, 2, 8, 9, 15, 16, 22, and 23). The effects of opioids were assessed 36 days after the first oxaliplatin administration, which is the optimal time for evaluation of chronic mechanical allodynia in this model. Allodynia-like behavior was evaluated using von-Frey monofilaments (pressure: 0.6, $1,1.4,2,4,6,8,10,15$, and $26 \mathrm{~g}$ ) at both sides of the paws and the values expressed as the sum of the paw withdrawals. The rats exhibiting a threshold change from $8-15 \mathrm{~g}$ before oxaliplatin injection to $<4 \mathrm{~g}$ at day 36 of oxaliplatin treatment were used in the experiments.

\section{Motor coordination}

Motor coordination was analyzed using a rota-rod test. FBC model animals were individually placed on the apparatus (KN75, Natsume Seisakusyo) rotating constantly at $15 \mathrm{rpm}$ and trained to continue walking on the rod for $2 \mathrm{~min}$. On the next day, mice were again placed on the rotating rod and animals that could keep walking for more than $4 \mathrm{~min}$ or a maximum of $5 \mathrm{~min}$ were chosen for drug analysis. After drug administration, mice were again placed on the rotating rod, and dropping time was measured for the 5-min duration of the test. Since a few mice jumped away from the rod soon after placing them on the rod, we have excluded those mice and used the mice that could have stayed on the rod for a moment. Results were calculated relative to the FBC saline-control groups.

\section{Statistical analyses}

The data are presented as the means \pm standard error of the mean (S.E.M). Statistical analyses were performed using GraphPad Prism 4.0 (GraphPad Software, San Diego, CA, USA). The significance of differences among groups was assessed by one-way analysis of variance (ANOVA). Differences between groups were analyzed using a Kruskal-Wallis test and Dunn's multiplecomparison test. Statistical analysis of differences between two groups was carried out with Student's $t$-test. In all analyses, $P<0.05$ was taken to indicate statistical significance.
Anti-nociceptive effects were expressed as pressure values $(\mathrm{g})$ or percentages of the maximal possible effect (MPE). MPE was calculated by $(\mathrm{g} 1-\mathrm{g} 0) \times 100 /$ ( $\mathrm{g} 2-\mathrm{g} 0$ ), where $\mathrm{g} 0$ is the mean of the paw withdrawal threshold in the FBC-saline group, g1 is the paw withdrawal threshold of FBC groups treated with drugs, and $\mathrm{g} 2$ is the mean of the paw withdrawal threshold in the sham-saline group.

\section{Results}

\section{Effects of opioids on the allodynia-like behaviors of FBC} model mice

Allodynia-like behaviors were detected at the ipsilateral paws of the FBC model mice at 14 days after tumor implantation with the lowest antinociceptive threshold of von-Frey monofilament (pressure: $0.008 \mathrm{~g}$ ) as described in our previous study (10). Using these mice, antinociceptive effects of oxycodone, morphine, tramadol, and tapentadol on allodynia-like behaviors were examined by the von-Frey monofilament test. Within the range of doses that do not induce behavior abnormality, subcutaneous (s.c.) administrations of oxycodone (1$10 \mathrm{mg} / \mathrm{kg}$, s.c.) and morphine $(5-50 \mathrm{mg} / \mathrm{kg}$, s.c. $)$ recovered the decreased paw withdrawal thresholds dose-dependently after 15 and $30 \mathrm{~min}$, respectively, with maximum paw withdrawal thresholds equipotent to the sham-treated groups $(110.82 \%$ in $10 \mathrm{mg} / \mathrm{kg}$ oxycodone and $80.52 \%$ in $50 \mathrm{mg} / \mathrm{kg}$ morphine, expressed as a percentages of the MPE) (Fig. 1). Although tramadol (10 $56 \mathrm{mg} / \mathrm{kg}$, s.c.) and tapentadol $(10-30 \mathrm{mg} / \mathrm{kg}$, s.c. $)$ dose-dependently recovered paw withdrawal thresholds after 30 and $15 \mathrm{~min}$, respectively, the next highest dose

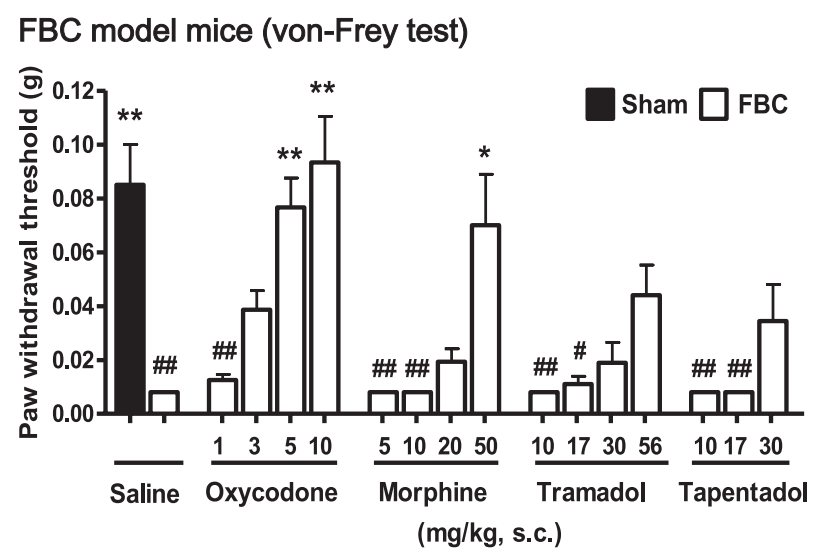

Fig. 1. Anti-nociceptive effects of opioids in the mouse FBC model. Allodynia-like behavior was measured by ipsilateral paw withdrawal using a series of von-Frey monofilaments. ${ }^{*} P<0.05,{ }^{*} P<0.01$ vs. the FBC-saline groups; ${ }^{\#} P<0.05,{ }^{\#} P<0.01$ vs. the sham-operated saline groups, $n=4-9$. Each value represents the mean \pm S.E.M. 
(100 mg/kg tramadol or $56 \mathrm{mg} / \mathrm{kg}$ tapentadol) caused behavior abnormality, such as convulsion and jumping which made it difficult to detect the threshold of those doses. Thus, anti-nociceptive thresholds of tramadol and tapentadol were restricted to a lower level than those of the sham-treated groups $(46.75 \%$ in $56 \mathrm{mg} / \mathrm{kg}$ tramadol and $34.41 \%$ in $30 \mathrm{mg} / \mathrm{kg}$ tapentadol by MPE). These results indicate that within the range of doses that do not induce abnormal behaviors, the dual-acting opioids tramadol and tapentadol possess distinct and restricted potencies compared with those of the MORselective opioids oxycodone and morphine in the FBC model.

\section{Effects of opioids on the motor coordination of FBC} model mice

Abnormalities identified at high doses of tramadol and tapentadol in the FBC model were further ascertained and compared with oxycodone and morphine by the rota-rod test, because this test is a major tool to quantify the effects of drugs on behaviors $(19,20)$. After 15and 30-min administrations, respectively, oxycodone $(100 \mathrm{mg} / \mathrm{kg}$, s.c. $)$ and morphine $(200 \mathrm{mg} / \mathrm{kg}$, s.c. $)$ markedly reduced the locomotor times on the rod compared with FBC saline-treated mice (Fig. 2). After 30- and 15 -min administrations, respectively, tramadol $(100 \mathrm{mg} /$ $\mathrm{kg}$ ) and tapentadol $(56 \mathrm{mg} / \mathrm{kg})$ also significantly reduced locomotor times. These rotating times were normalized to the FBC saline-control groups and expressed as percentages.

The anti-nociceptive doses examined in Fig. 1 were normalized to MPE and added to the line graph in Fig. 2 to compare with the doses used in the rota-rod analyses. The dissociation ratio of anti-nociception and motor coordination was calculated from the $\mathrm{ED}_{80}$ value of the anti-nociceptive doses and the no-observed-adverse-effect level (NOAEL) dose, respectively. A high NOAEL/ anti-nociceptive dose was detected for oxycodone (10-fold) and morphine (2-fold). For tramadol and tapentadol, the anti-nociceptive doses estimated to exert sham-threshold levels were greater than the doses of NOAEL with a ratio of less than one-fold. Accordingly, the safety margin of the MOR-selective opioids oxycodone and morphine and the dual-acting opioids tramadol and tapentadol are different, suggesting these two groups of opioids have distinct mechanisms of action.

Effects of MOR, $\alpha_{2}$-adrenoceptor, or $\alpha_{1}$-adrenoceptor antagonists on anti-nociception and motor coordination of oxycodone and tapentadol in FBC model mice

In addition to their MOR actions, tramadol and tapentadol induce synaptic NE by inhibiting NET $(3,21,22)$. It has been suggested that the anti-nociceptive effects of tapentadol and tramadol are synergistically regulated by $\alpha_{2}$-adrenoceptors and MORs and highly inhibited by $\alpha_{2}$-adrenoceptor antagonists in several model animals, such as the DPN model and spinal nerve ligation model $(3,14,23)$. To determine whether the anti-nociceptive effects of the dual-acting agonists in our FBC model are mediated by these mechanisms, we compared oxycodone and tapentadol. The opioids were chosen because oxycodone has greater anti-nociception than morphine in this model and tramadol itself does not act on MOR. We examined anti-nociceptive effects in the FBC model by pre-administration of a MOR antagonist ( $\beta$-FNA, 25 $\mathrm{mg} / \mathrm{kg}$, s.c.), an $\alpha_{2}$-adrenoceptor antagonist (yohimbine, $2 \mathrm{mg} / \mathrm{kg}$, s.c.), or an $\alpha_{1}$-adrenoceptor antagonist (prazosin, $1 \mathrm{mg} / \mathrm{kg}$, s.c.). Before the examination, we confirmed that the antagonists themselves had no effects on nociceptive and anti-nociceptive thresholds of the mice (data not shown). The doses of opioids were chosen those for the highest thresholds shown in Fig. 1 (10 mg/kg oxycodone or $30 \mathrm{mg} / \mathrm{kg}$ tapentadol, s.c.). After $24 \mathrm{~h}, 5$ - and 30 -min pre-administration of antagonists, respectively,

\section{FBC model mice (Rota-rod test, von-Frey test)}

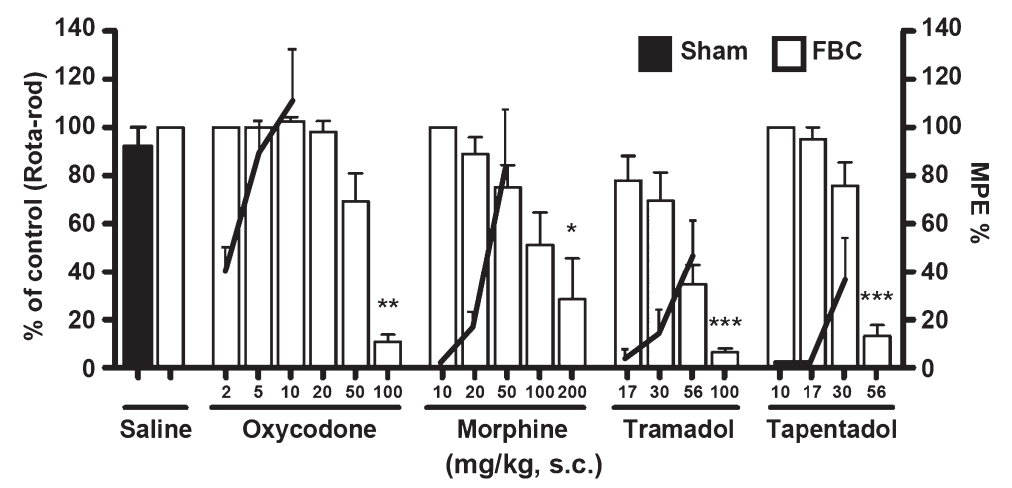

Fig. 2. Effect of opioids on motor coordination in the mouse FBC model. Dose-response effects of each agonist during the rota-rod tests are shown in the left $\mathrm{Y}$-axis with bar graph. The rotating times were normalized to the FBC saline-control groups and expressed as percentages. The anti-nociceptive effect examined in Fig. 1 is shown in the right $\mathrm{Y}$-axis with the line graph expressed as MPE percentage. $* P<0.05, * * P<0.01, * * * P<0.001$ vs. the FBC saline-treated groups, $n=5-9$. Each value represents the mean \pm S.E.M. 


\section{(A) FBC model mice (von-Frey test)}

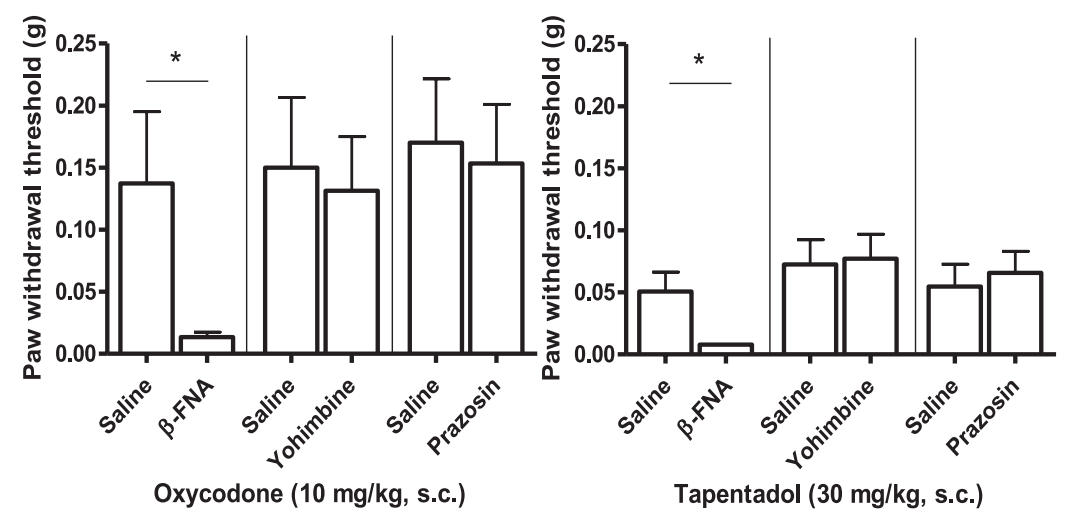

(B) FBC model mice (Rota-rod test)
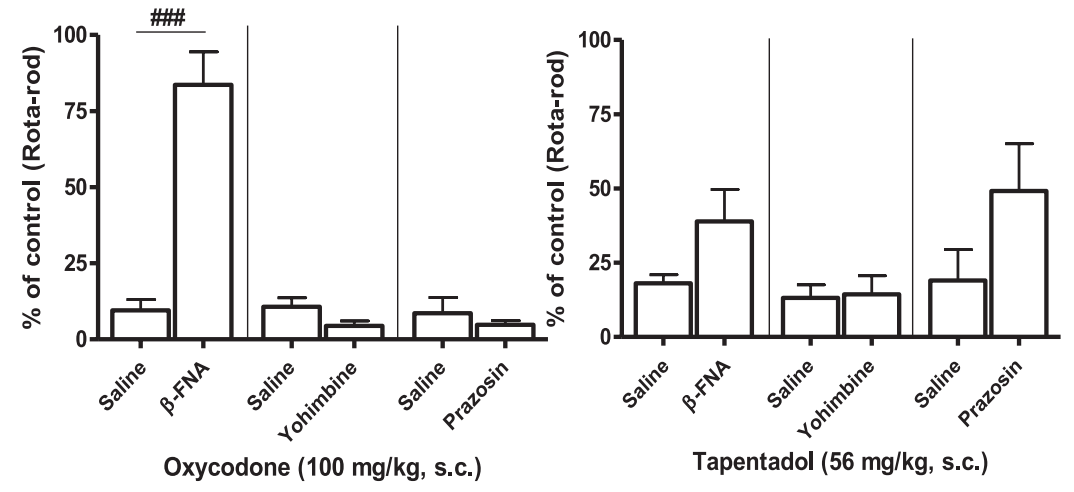

Fig. 3. Mechanisms involved in oxycodone- and tapentadol-induced anti-nociception and motor impairment in the FBC models, as determined by blockade of MOR ( $\beta$-FNA), $\alpha_{1}$-adrenoceptor (prazosin), or $\alpha_{2}$ adrenoceptor (yohimbine) activity. A) Effect of each antagonist on anti-nociception. Allodynia-like behavior was measured by ipsilateral paw withdrawal using a series of von-Frey monofilaments, $n=8-11$. B) Effect of each antagonist on motor impairment of the agonists, examined by rota-rod, $\mathrm{n}=7-9 .{ }^{*} P<0.05,{ }^{\# \#} P<0.001$ vs. the saline-controlled groups for each antagonist. Each value represents the mean \pm S.E.M. the anti-nociceptive effects of oxycodone and tapentadol were significantly reduced in $\beta$-FNA pre-treated mice $(89.76 \%$ or $84.22 \%$ reduction in oxycodone or tapentadol, respectively, vs. saline-control group), whereas no effects of yohimbine and prazosin were detected compared with saline-control animals examined at the same timing for each antagonist (Fig. 3A). Therefore, these results suggested the anti-nociceptive effects of oxycodone and tapentadol are regulated by MOR activation.

Again using oxycodone and tapentadol, we examined the mediating factors that induce motor coordination dysfunction using doses determined in Fig. $2(100 \mathrm{mg} / \mathrm{kg}$ oxycodone or $56 \mathrm{mg} / \mathrm{kg}$ tapentadol, s.c.). After 24-h, 5-min, and 30-min pre-administration of antagonists, respectively, motor impairment of oxycodone was reduced significantly by $\beta$-FNA $(25 \mathrm{mg} / \mathrm{kg}$, s.c.) but not by yohimbine $(2 \mathrm{mg} / \mathrm{kg}$, s.c.) and prazosin $(1 \mathrm{mg} / \mathrm{kg}$, s.c.) (Fig. 3B). In contrast, impaired coordination of tapentadol was partially recovered by $\beta$-FNA $(20.84 \%$ improvement vs. saline-control group) and prazosin (28.12\% improvement vs. saline-control group), while the statistical significance was not detected in both antagonists (Fig. 3B). Similar to oxycodone, yohimbine showed no effect (Fig. 3B). According to these results, the factors that mediate motor impairment seemed different between selective MOR agonists and dualacting opioids.

Effects of oxycodone and tapentadol on the allodynialike behaviors of two neuropathic pain models

Although several opioids such as oxycodone have been well characterized for the treatment of neuropathic pain, it has been reported that neuropathic pain is more difficult to treat with opioids compared to nociceptive and inflammatory pain in clinical and non-clinical settings $(10,24,25)$. In the FBC model, tapentadol had a lesser effect on allodynia-like behaviors (Fig. 1). Thus, we focused on neuropathy and examined the anti-nociceptive effects of tapentadol and oxycodone using specific neuropathic pain models. We chose the pSNL model rats because it is a well-characterized specific neuropathic pain model with no reports on the effects of tapentadol.

We first examined the effective doses of oxycodone and tapentadol because rats may have different sensitivity 


\section{Partial sciatic nerve ligation ( $\mathrm{pSNL}$ ) model rats (von-Frey test)}

(A)

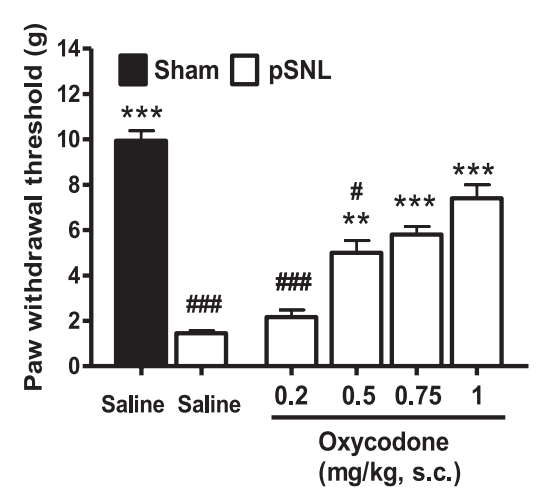

(B)

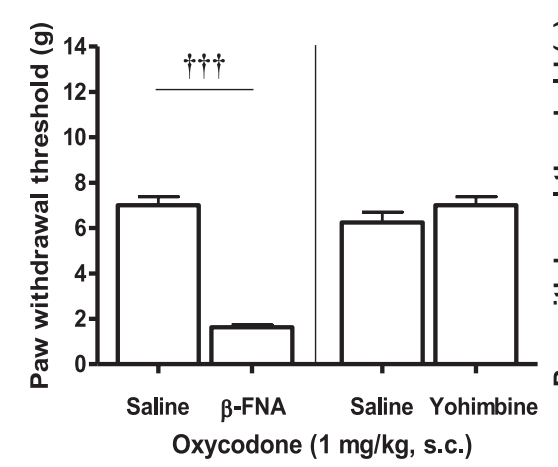

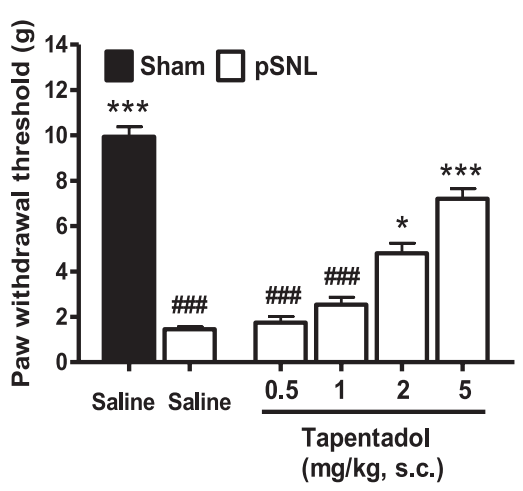

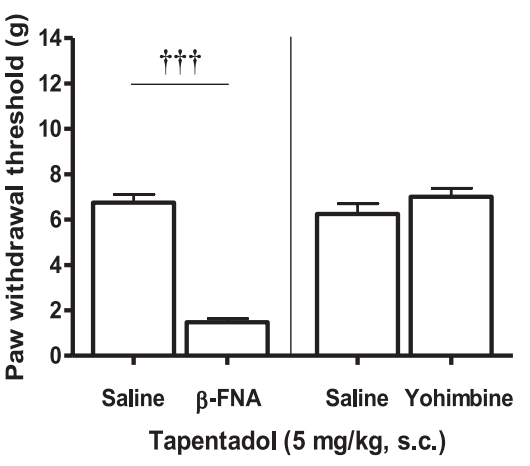

Fig. 4. Anti-nociceptive effects of oxycodone and tapentadol on allodynia-like behaviors in the rat partial spinal nerve ligation ( $\mathrm{pSNL}$ ) model. A) Dose-response effects, $\mathrm{n}=10-14$. B) Effects of a MOR antagonist ( $\beta$-FNA) or $\alpha_{2}$-adrenoceptor antagonist (yohimbine) on the anti-nociceptive effect of the agonists, $\mathrm{n}=8$. Allodynia-like behavior was measured by ipsilateral paw withdrawal using a series of von-Frey monofilaments. $* P<0.05, \quad * * P<0.01, \quad * * * P<0.001 \quad$ vs. pSNL-saline groups; ${ }^{\#} P<0.05$, ${ }^{\# \# \#} P<0.001$ vs. the sham-operated saline groups. ${ }^{\dagger \dagger} P<0.001$ vs. the saline-controlled groups for each antagonist. Each value represents the mean \pm S.E.M.

to opioids than mice $(2,26)$. Within the range of doses that does not induce sedation, s.c. administrations of oxycodone $(0.2-1 \mathrm{mg} / \mathrm{kg})$ and tapentadol $(0.5-5 \mathrm{mg} / \mathrm{kg})$ dose-dependently and significantly recovered the decreased paw withdrawal thresholds of pSNL model rats after 15- and 30-min administrations, respectively, with maximal thresholds equipotent as the sham-treated paws $(70.15 \%$ in $1 \mathrm{mg} / \mathrm{kg}$ oxycodone and $67.79 \%$ in 5 $\mathrm{mg} / \mathrm{kg}$ tapentadol by MPE) (Fig. 4A). We next examined the components involved in the anti-nociceptive mechanisms. The $24-\mathrm{h}$ pre-administration of $25 \mathrm{mg} / \mathrm{kg} \beta$-FNA (s.c.) significantly and completely decreased paw withdrawal thresholds in both the oxycodone $(1 \mathrm{mg} / \mathrm{kg}$, s.c.) and tapentadol $(5 \mathrm{mg} / \mathrm{kg}$, s.c.) groups $(76.79 \%$ or $78.15 \%$ reduction in oxycodone or tapentadol, respectively, vs saline-control group). Although yohimbine is reported to decrease anti-nociception of tapentadol in some pain models, $2 \mathrm{mg} / \mathrm{kg}$ (s.c.) pre-administration had no effect on both agonists. These results indicate that oxycodone and tapentadol possess similar profiles mediated by MORs in the pSNL neuropathic pain model (Fig. 4B).

We also used the oxaliplatin neuropathic pain model rats to examine the anti-nociceptive effects of oxycodone and tapentadol using the von-Frey test. Subcutaneous administrations of oxycodone $(0.1-1 \mathrm{mg} / \mathrm{kg})$, which did not induce sedation, dose-dependently recovered the decreased paw withdrawal thresholds after $15 \mathrm{~min}$ of administration and the maximal paw withdrawal thresholds were equipotent to those of the sham-treated group $(83.64 \%$ or $132.24 \%$ in 0.56 or $1 \mathrm{mg} / \mathrm{kg}$ oxycodone respectively, by MPE) (Fig. 5A). In contrast, although the highest dose of tapentadol $(5 \mathrm{mg} / \mathrm{kg})$ recovered paw withdrawal threshold after 30-min administration compared with the oxaliplatin-saline group, those thresholds were lower than that of the sham-saline group (53.27\% in $5 \mathrm{mg} / \mathrm{kg}$ tapentadol by MPE). Since more than $5 \mathrm{mg} / \mathrm{kg}$ induced sedation, the anti-nociceptive effect of tapentadol was found to be lower than that of oxycodone within the range of doses without abnormal behavior in this model.

The mechanisms involved in anti-nociception of these opioids were also compared in the oxaliplatin model rats. The 24 -h pre-administration of $\beta$-FNA (10 $\mathrm{mg} / \mathrm{kg}$, s.c.) significantly decreased the paw withdrawal thresholds of oxycodone $(0.56 \mathrm{mg} / \mathrm{kg}$, s.c.) and tapentadol $(5 \mathrm{mg} / \mathrm{kg}$, s.c.) $(71.39 \%$ or $53.45 \%$ reduction in oxycodone or tapentadol, respectively). In addition, although pre-administration of yohimbine ( $2 \mathrm{mg} / \mathrm{kg}$, s.c.) 


\section{Oxaliplatin model rats (von-Frey test)}

(A)

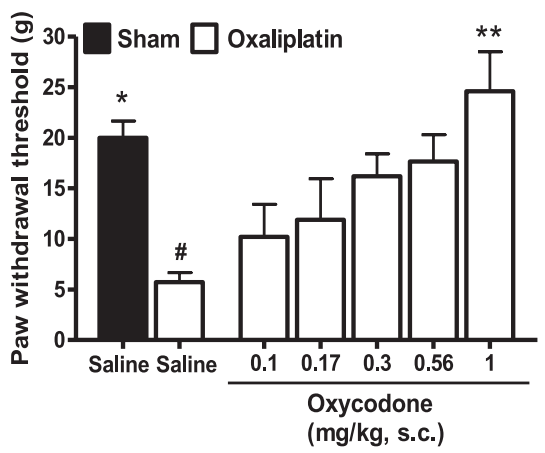

(B)
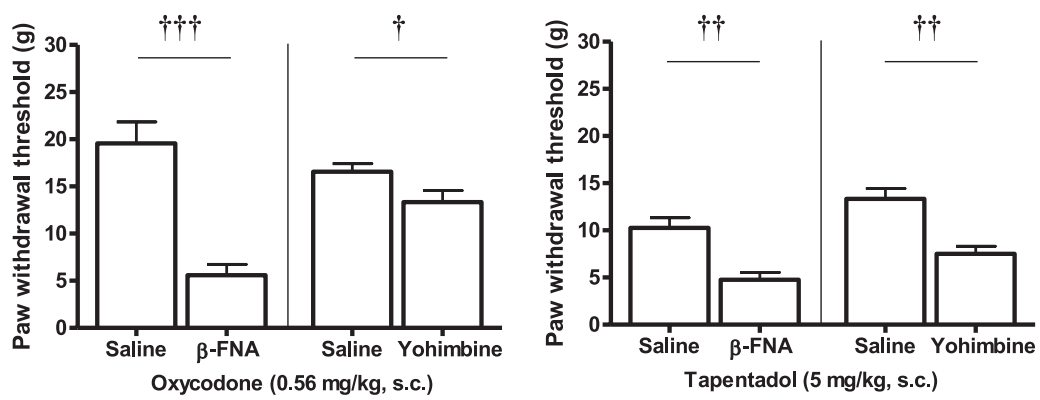

Fig. 5. Anti-nociceptive effects of oxycodone and tapentadol on allodynia-like behaviors in the rat oxaliplatin-induced neuropathy model. A) Doseresponse effects of the agonists, $\mathrm{n}=5-10$. B) Effects of the MOR antagonist ( $\beta$-FNA) or $\alpha_{2}$-adrenoceptor antagonist (yohimbine) on anti-nociceptive effects, $\mathrm{n}=6-8$. Allodynia-like behaviors are expressed as the sum of the right and left paw withdrawal thresholds using von-Frey monofilaments. $* P<0.05, * * P<0.01$ vs. the oxaliplatin-saline-treated groups; ${ }^{\#} P<0.05$, ${ }^{\#} P<0.01$ vs. the sham-treated saline groups. ${ }^{\dagger} P<0.05$, ${ }^{\dagger} P<0.01,{ }^{\dagger \dagger} P<0.001$ vs. the saline-control groups for each antagonist. Each value represents the mean \pm S.E.M. modestly decreased the threshold of oxycodone, it markedly decreased that of tapentadol $(19.55 \%$ or $43.74 \%$ reduction in oxycodone or tapentadol, respectively) (Fig. 5B). These results indicate that oxycodone and tapentadol possess distinct anti-nociceptive profiles with different levels of efficacy and different antinociceptive mechanisms.

Based on the results from these two models, the potency of the dual-acting opioid in the oxaliplatin model was similar to that in the FBC model. However, in the pSNL model the dual-acting opioid had greater potency, indicating that the potency of the dual-acting opioids might change with the type of pain. Therefore, the lower potency of the dual-acting opioids observed in the FBC model was not due to decreased anti-nociception in neuropathic pain.

\section{Discussion}

In this study, we used a FBC model of bone cancer pain to examine whether the NET inhibiting activity of the dual-acting opioids (tramadol and tapentadol) affect antinociception of MOR by comparing their potency with the clinically used MOR-selective opioids (oxycodone and morphine). Although we found potent anti-nociceptive effects in MOR-selective opioids with similar thresholds to the sham-treated groups, the thresholds of dual-acting opioids were lower. In addition, a difference in abnormal behaviors in the FBC model was detected at the antinociceptive doses of MOR-selective and dual-acting opioids, which may be caused by differences in NET inhibition.

Rota-rod studies have revealed that the safety margins of oxycodone and morphine are wider than those of tramadol and tapentadol. We identified the distinct mechanism involved in abnormal behaviors of these agonists since the MOR antagonist fully recovered the rotating time of oxycodone-treated animals, while not only the MOR antagonist but also the $\alpha_{1}$-adrenoceptor antagonist partially and similarly recovered the rotating time in tapentadol-treated animals. We assumed that the narrow safety margin of the dual-acting agonists might be caused by additional effect of NET inhibition, which increases synaptic NE to activate $\alpha_{1}$-adrenoceptors and influence motor activities. In support of this, it has been reported that $\alpha_{1}$-adrenoceptor, but not $\alpha_{2}$-adrenoceptor, agonists induce motor and autonomic disturbances (27, 28). In addition, microdialysis studies have shown that tapentadol and tramadol increase NE release at synapses, whereas morphine decreases basal NE release at spinal 
and supraspinal sites $(3,19,22,29)$. Although no reports on oxycodone are available, it seems that the contribution of the $\alpha_{1}$-adrenoceptor-mediated pathway to motor function differs between MOR-selective and dual-acting opioids.

In studies of the anti-nociceptive mechanisms in the FBC model, we found that tapentadol-induced antinociception is mediated by MOR activation. This may indicate that NET inhibiting function does not contribute to tapentadol-induced anti-nociception in this model. In contrast to our results, it was reported previously that anti-nociception of tapentadol was caused by the synergistic or additive actions of MOR and $\alpha_{2}$-adrenoceptor activation in several models of neuropathic, nociceptive, and inflammatory pain $(3,12,23)$. Therefore, we confirmed whether the direct activation of $\alpha_{2}$-adrenoceptors by clonidine could induce anti-nociception in the FBC model. We found that a dose of clonidine that was effective on the tail-flick test $(0.3 \mathrm{mg} / \mathrm{kg}$, s.c.) caused only a slight anti-nociceptive effect in the FBC model with no significant difference compared to the saline-treated groups (30), which indicated the low antinociceptive potency of the $\alpha_{2}$-adrenoceptor-mediated pathways. We should note that $0.5 \mathrm{mg} / \mathrm{kg}$ clonidine caused a significant drop from the rota-rod (data not shown), similar to was has been described in normal mice (31). Accordingly, a pathway that inhibits NET appears insufficient to contribute to anti-nociception in the FBC model.

In studies focusing on MOR activity, the differences in agonist potencies between MOR-selective opioids and dual-acting opioids might be due to changes in MOR expression in the FBC model and the degree of agonist activities. Compared with sham controls, we have determined previously that the expression of MORs on the cell membrane in the FBC model was reduced by approximately $30 \%$, determined by $\mathrm{B}_{\max }$ of ${ }^{3} \mathrm{H}$ DAMGO binding, without affecting the $\mathrm{K}_{\mathrm{d}}$ in pain-related regions (32). In addition, it has been reported that the agonist activity of partial agonists was reduced more by a change in the number of receptors compared to that of full agonists $(33,34)$. Although the agonist activities of the opioids examined here were not compared directly, a previous report showed that the maximum responses of oxycodone and morphine were similar with more than $95 \%$ in ${ }^{35} \mathrm{SGTP} \gamma \mathrm{S}$ binding relative to that obtained for DAMGO (35). In contrast, tapentadol and the M1 metabolite of tramadol exhibit lower maximum responses than morphine $(3,36)$. In these respects, tapentadol and the M1 metabolite of tramadol are assumed to be partial agonists for MOR compared with oxycodone and morphine, which are full agonists for MOR. Thus, a reduction of the receptor levels in the FBC model would be expected to influence their potencies for MOR activation.

It has been reported that tapentadol synergistically exhibit its anti-nociceptive effect primarily via MOR in a tail-flick test and a complete Freund's adjuvant (CFA) model, whereas it synergistically exhibited its antinociceptive effect primarily via $\alpha_{2}$-adrenoceptors in the DPN, carrageenan, and spinal nerve ligation models (3, $12,14,23)$. With our results in the pSNL and oxaliplatin models, it was assumed that tapentadol had different anti-nociceptive potencies, as well as anti-nociceptive mechanisms, dependent on the pain model used in the study. The differences in NET inhibition may be related to differences in NE content in these models. For example, our preliminary data by high-performance liquid chromatography (HPLC) has shown that NE content in the dorsal horn is down-regulated $60 \%$ in the pSNL model at the time of our anti-nociceptive examination 14 days after surgery (data not shown). In the spinal nerve ligation model, however, NE in the spinal dorsal horn increased $126 \% 10$ days after surgery (37). Furthermore, a recent study showed that systemic administration of tapentadol elevated cerebral spinal fluid NE in sham and spinal nerve ligated animals, and NE remained elevated for $90 \mathrm{~min}$ in the spinal nerve ligated animals only, suggesting sensitization of the NE neurons in this model (29). Even though NE was not examined in all related pain models, differences in NE content might influence the level of NE and NET inhibition; in the pSNL, low NE might be insufficient for NE release and leads to less NET inhibition. Based on these ideas, further studies are needed to determine why tapentadol contributes differently to MOR and NET inhibition in different pain models.

In conclusion, although the dual-acting opioids may have potency for some neuropathic and inflammatory pain conditions, we found that they have low potency against bone cancer pain. This might be caused by the lack of a contribution by the $\alpha_{2}$-adrenoceptor-mediated pathway to anti-nociception, although the $\alpha_{1}$-adrenoceptor may be involved in motor impairment. Therefore, we conclude that the additional effect of NET inhibition on MOR activation by the dual-acting opioids does not contribute to anti-nociception in bone cancer pain. Although we should further consider these dual-acting opioids for their potency in other cancer pain conditions, these studies may offer one of the pharmacological characterizations of the dual acting opioids.

\section{Acknowledgment}

We would like to thank Mr. Ryuta Tamano for his technical support and advice. 


\section{Conflicts of Interest}

H. Ono, A. Nakamura, T. Kanbara, K. Minami, S. Shinohara, G. Sakaguchi, and T. Kanemasa are employees of Shionogi Co., Ltd., the manufacturer of oxycodone and morphine. No funding is associated with this paper.

\section{References}

1 Hennis HH, Friderichs E, Schneider J. Receptor binding, analgesic and antitussive property of tramadol and other selective opioids. Arznemittelforschung. 1988;38:877-880.

2 Raffa RB, Friderichs E, Reimann W, Shank RP, Codd EE, Vaught JL. Opioid and nonopioid components independently contribute to the mechanism of action of tramadol an 'atypical' opioid analgesic. J Pharmacol Exp Ther. 1992;260:275-285.

3 Tzschentke TM, Christoph T, Kögel B, Schiene K, Hennies $\mathrm{HH}$, Englberger W, et al. (-)-(1R,2R)-3-(3-dimethylamino-1ethyl-2-methyl-propyl)-phenol hydrochloride (tapentadol $\mathrm{HCl}$ ): a novel mu-opioid receptor agonist/norepinephrine reuptake inhibitor with broad-spectrum analgesic properties. J Pharmacol Exp Ther. 2007;323:265-276.

4 Mercadante S, Arcuri E. Breakthrough pain in cancer paitients: pathophysiology and treatment. Cancer Treat Rev. 1998;24: 425-432.

5 Portenoy RK, Payne D, Jacobsen P. Breakthrough pain: characteristics and impact in patients with cancer pain. Pain. 1999;81: 129-134.

6 Matza LS, Fallowfield LJ, Chung KC, Currie BM, Van Brunt K, Patrick DL. Patient-reported outcome instruments used to assess pain and functioning in studies of bisphosphonate treatment for bone metastases. Support Care Cancer. 2012;20: 657-677.

7 Komiya S, Zenmyo M, Inoue A. Bone tumors in the pelvis presenting growth during pregnancy. Arch Orthop Trauma Surg. 1999;119:22-29.

8 Honore P, Lugar NM, Sabino MAC, Schwei MJ, Rogers SD, Mash DB, et al. Osteroprotegerin blocks bone cancer-induced skeletal destruction, skeletal pain and pain-induced neurochemical reorganization of the spinal cord. Nat Med. 2000;6: 521-528.

9 Pandit-Taskar N, Batraki M, Divgi CR. Radiopharmaceutical therapy for palliation of bone pain from osseous metastases. J Nucl Med. 2004;45:1358-1365.

10 Minami K, Hasegawa M, Ito $\mathrm{H}$, Nakamura A, Tomii $\mathrm{T}$, Matsumoto M, et al. Morphine, oxycodone, and fentanyl exhibit different analgesic profiles in mouse pain models. J Pharmacol Sci. 2009;111:60-72.

11 Kayser V, Besson JM, Guilbaud G. Evidence for a noradrenergic component in the antinociceptive effect of the analgesic agent tramadol in an animal model of clinical pain, the arthritic rat. Eur J Pharmacol. 1992;224:83-88.

12 Schiene K, De Vry J, Tzschentke TM. Antinociceptive and antihyperalgesic effects of tapentadol in animal models of inflammatory pain. J Pharmacol Exp Ther. 2011;339:537-544.

13 Narita M, Nakamura A, Ozaki M, Imai S, Miyoshi K, Suzuki M, et al. Comparative pharmacological profiles of morphine and oxycodone under a neuropathic pain-like state in mice: evidence for less sensitivity to morphine. Neuropsychopharmacology. 2008;33:1097-1112.
14 Schröder W, Tzschentke TM, Terlinden R, De Vry J, Jahnel U, Christoph T, et al. Synergistic interaction between the two mechanisms of action of tapentadol in analgesia. J Pharmacol Exp Ther. 2011;337:312-320.

15 Horiguchi N, Ago Y, Asada K, Kita Y, Hiramatsu N, Takuma K, et al. Involvement of spinal 5-HT1A receptors in isolation rearing-induced hypoalgesia in mice. Psychopharmacology (Berl). 2013;227:251-261.

16 Seltzer Z, Dubner R, Shir Y. A novel behavioral model of neuropathic pain disorders produced in rats by partial sciatic nerve injury. Pain. 1990;43:205-218.

17 Malmberg AB, Basbaum AI. Partial sciatic nerve injury in the mouse as a model of neuropathic pain: behavioral and neuroanatomical correlates. Pain. 1998;76:215-222.

18 Sakurai M, Egashira N, Kawashiri T, Yano T, Ikesue H, Oishi R. Oxaliplatin-induced neuropathy in the rat: involvement of oxalate in cold hyperalgesia but not mechanical allodynia. Pain. 2009;147:165-174.

19 Simiand J, Keane PE, Biziere K, Soubrie P. Comparative study in mice of tetrazepam and other centrally active skeletal muscle relaxants. Arch Int Pharmacodyn Ther. 1989;297:272-285.

20 Farkas S, Berzsenyi P, Kárpáti E, Kocsis P, Tarnawa I. Simple pharmacological test battery to assess efficacy and side effect profile of centrally acting muscle relaxant drugs. J Pharmacol Toxicol Methods. 2005;52:264-273.

21 Tzschentke TM, Folgering JH, Flik G, De Vry J. Tapentadol increases levels of noradrenaline in the rat spinal cord as measured by in vivo microdialysis. Neurosci Lett. 2012;507: 151-155.

22 Bloms-Funke P, Dremencov E, Cremers TI, Tzschentke TM. Tramadol increases extracellular levels of serotonin and noradrenaline as measured by in vivo microdialysis in the ventral hippocampus of freely-moving rats. Neurosci Lett. 2011;490: 191-195.

23 Kögel B, De Vry J, Tzschentke TM, Christoph T. The antinociceptive and antihyperalgesic effect of tapentadol is partially retained in OPRM1 ( $\mu$-opioid receptor) knockout mice. Neurosci Lett. 2011;491:104-107.

24 Cherny NI, Thaler HT, Friedlander-Klar H, Lapin J, Foley KM, Houde R, et al. Opioid responsiveness of cancer pain syndromes caused by neuropathic or nociceptive mechanisms: a combined analysis of controlled, single-dose studies. Neurology. 1994;44: 857-861.

25 Baron R, Binder A, Wasner G. Neuropathic pain: diagnosis, pathophysiological mechanisms, and treatment. Lancet Neurol. 2010;9:807-819.

26 Hayes AG, Sheehan MJ, Tyers MB. Differential sensitivity of models of antinociception in the rat, mouse and guinea-pig to mu- and kappa-opioid receptor agonists. Br J Pharmacol. 1987; 91:823-832.

27 Howe JR, Wang JY, Yaksh TL. Selective antagonism of the antinociceptive effect of intrathecally applied alpha adrenergic agonists by intrathecal prazosin and intrathecal yohimbine. J Pharmacol Exp Ther. 1983;224:552-558.

28 Wada T, Hasegawa Y, Ono H. Characterization of alpha1adrenoceptor subtypes in facilitation of rat spinal motoneuron activity. Eur J Pharmacol. 1997;340:45-52.

29 Meske DS, Xie JY, Oyarzo J, Badghisi H, Ossipov MH, Porreca F. Opioid and noradrenergic contributions of tapentadol in experimental neuropathic pain. Neurosci Lett. 2013. In press. 
30 de Moura RS, Rios AA, Santos EJ, Nascimento AB, de Castro Resende A, Neto ML, et al. Role of the NO-cGMP pathway in the systemic antinociceptive effect of clonidine in rats and mice. Pharmacol Biochem Behav. 2004;78:247-253.

31 Dogrul A, Uzbay IT. Topical clonidine antinociception. Pain. 2004;111:385-391.

32 Nakamura A, Hasegawa M, Minami K, Kanbara T, Tomii T, Nishiyori A, et al. Differential activation of the $\mu$-opioid receptor by oxycodone and morphine in pain-related brain regions in a bone cancer pain model. Br J Pharmacol. 2013;168:375-388.

33 Cordeaux Y, Briddon SJ, Megson AE, Mcdonnell J, Dickenson J. Influence of receptor number on functional responses elicited by agonists acting at the human adenosine A1 receptor: evidence for signalling pathway-dependent changes in agonist potency and relative intrinsic activity. Mol Pharmacol. 2000;58:10752000.

34 McDonald J, Barnes TA, Okawa H, Williams J, Calo G,
Rowbotham DJ, et al. Partial agonist behaviour depends upon the level of nociceptin/orphanin FQ receptor expression: studies using the ecdysone-inducuble mammalian expression system. Br J Pharmacol. 2003;140:61-70.

35 McPherson J, Rivero G, Baptist M, Llorente J, Al-Sabah S, Krasel C, et al. $\mu$-opioid receptors: correlation of agonist efficacy for signalling with ability to activate internalization. Mol Pharmacol. 2010;78:756-766.

36 Gillen C, Haurand M, Kobelt DJ, Wnendt S. Affinity, potency and efficacy of tramadol and its metabolites at the cloned human mu-opioid receptor. Naunyn Schmiedebergs Arch Pharmacol. 2000;362:116-121.

37 Hayashida K, Clayton BA, Johnson JE, Eisenach JC. Brain derived nerve growth factor induces spinal noradrenergic fiber sprouting and enhances clonidine analgesia following nerve injury in rats. Pain. 2008;136:348-355. 